\title{
Improving clinic attendances for core medical trainees at a busy district general hospital
}

\author{
Authors: Pooja Mithani and Leila Bafadhel
}

\section{Introduction}

Attending clinics is large part of completing the core medical training competencies. The minimum number of clinics required has now increased from 12 to 20 per annum. Core medical trainees (CMTs) often struggle to attend owing to busy medical wards, oncalls and staff shortages. This quality improvement project aimed to improve attendance with simple measures.

\section{Methods}

We collected data to assess the number of CMTs attending clinic during their time off. A clinic rota was devised which allocated weekly afternoon clinic sessions for trainees. The first Plan, Do, Study, Act (PDSA) cycle included uploading the clinic rota onto the online on-call roster. The second PDSA cycle consisted of sending out clinic timetables for each specialty. The third PDSA cycle involved sending out more detailed timetables, including named consultants and themes of the clinic, to provide trainees with choice. We collated data by sending anonymous surveys to ten CMTs. We monitored the percentage of trainees who were attending clinics in their own time and how many were on track to meet their Annual Review of Competency Progression (ARCP) requirements.

\section{Results and discussion}

Initial data suggested that $70 \%$ of trainees had to come in on off days and during annual leave to complete their clinics.

Following the first PDSA cycle, $57 \%$ had seen an improvement in their attendances, with $14 \%$ attending all their allocated clinic sessions. The second PDSA cycle showed $25 \%$ of trainees had attended all their allocated clinics and that $100 \%$ of them found an improvement in being able to organise which clinic to attend. None of the CMTs attended clinics in their own time and $88 \%$ of trainees were on target to meet their ARCP requirements. Seventyfive per cent of trainees found ward duties the biggest barrier to attending clinics.

Authors: Southend University Hospital NHS Foundation Trust, Essex, UK
Having a given day of the week set in advance for clinic meant that arrangements could be made to provide adequate cover on the wards.

Access to clinic schedules makes organising attendance much more efficient. Ward duties remained the biggest hurdle to obtaining mandatory attendances.

\section{Conclusion}

Allocating clinic afternoons on the online on-call roster eliminated the scheduling constraint of fitting in clinics around daily duties. The implementation of the rota provided an overall improvement in the clinic attendances. 